\title{
CORRESPONDENCE
}

\section{Challenging an exercise challenge protocol}

\section{To the Editor:}

In an interesting study, published in The Journal, HABY et al. [1] compared an exercise challenge test with histamine challenge in children. Some children responded to both challenges, and some reacted to one challenge but not to the other. Most of the children reacted to neither challenge. I agree with at least one of the conclusions, namely that the study "suggests that the two challenges identify different abnormalities of the airways".

On the other hand, I object to the way in which the authors presented the "relationship between dose-response ratio to histamine and the percentage fall in forced expiratory volume in one second $\left(\mathrm{FEV}_{1}\right)$ to exercise, shown with the regression line" in their figure 2 . The regression line gives the uncritical reader the impression that there is a significant correlation, with a slope of about $45^{\circ} \mathrm{C}$. There are at least two fallacies in this presentation. Firstly, for 91 of the 94 subjects there is no obvious correlation, at least not in agreement with the regression line presented. Thus, the regression, if any, cannot be a linear one. Secondly, the three out of 94 subjects which cause the "slope" had more than a $40 \%$ fall in $\mathrm{FEV}_{1}$ on exercise. From the Method section, it is clear that in these three "the postexercise measurements were stopped (before the $10 \mathrm{~min}$ value) and 200 $\mu \mathrm{g}$ salbutamol aerosol administered". Thus, the recorded postexercise values for these three subjects were not true maximal values, and this will distort the "slope" still further.

My own conclusion is that their figure 2 does not show any physiological correlation between the two types of tests for bronchial hyperreactivity - in spite of the "statistical significance". The figure demonstrates that the computer will draw the "best possible" straight line when ordered to do so, even when there is no true linear regression.

\section{E. Berglund}

Renströmska Sjukhuset, S-402 64 Göteborg, Sweden.

\section{Reference}

1. Haby MM, Anderson SD, Peat JK, Mellis CM, Toelle BG, Woolcock AJ. An exercise challenge protocol for epidemiological studies of asthma in children: comparison with histamine challenge. Eur Respir J 1994; 7: $43-49$.

\section{$\underline{R E P L Y}$}

\section{From the authors:}

We thank E. Berglund for his interest in our paper. He draws attention to the difficulty of comparing two tests with different units of measurement. We used Pearson's correlation coefficient and linear regression to compare two tests of bronchial provocation.

In response to E. Berglund's first comment, we accept that the three children with a fall in forced expiratory volume in one second $\left(\mathrm{FEV}_{1}\right)$ greater than $40 \%$ had a large influence on the regression line. We did not exclude these children from the analysis because the extreme response could not be traced to measurement error. However, when these three children are excluded from the analysis, the correlation remained significant $(r=$ $0.28 ; \mathrm{p}=0.008$ ), although the relationship is weaker than when the three children are included ( $\mathrm{r}=0.65 ; \mathrm{p}=0.0001)$. Recently, we have extended the sample size to 201 children and the correlation in this larger sample is 0.59 , $\mathrm{p}=0.0001$, with the regression line following a similar slope to that in figure 2 .

In response to the second comment, both challenges

have a cut-off point after which testing is discontinued (to prevent severe airway narrowing). The histamine challenge was stopped when the fall in $\mathrm{FEV}_{1}$ was greater than $20 \%$, after exercise challenge bronchodilator was given if the $\mathrm{FEV}_{1}$ had fallen by $40 \%$ at any time. The figure, therefore, presents the responses to the two challenges using the methods described and not "true maximal values".

We believe it is important to include the figure because it gives the reader more information about the relationship between the responses to the two challenges than can be conveyed by table 1 . The figure and regression line show that there is some relationship between the two challenges, even with the three extreme values excluded. However, the exact nature of the abnormality identified by each challenge and the mechanism by which the airways narrow is yet to be determined.

M.M. Haby*, S.D. Anderson**, J.K. Peat*, C.M. Mellis $^{+}$, B.G. Toelle*, A.J. Woolcock*

*Department of Medicine, University of Sydney, NSW 2006, Australia, **Dept of Respiratory Medicine, and +Epidemiology Unit, Institute of Respiratory Medicine, Royal Prince Alfred Hospital, NSW 2050, Australia. 